\title{
AGUSTÍN Y CICERÓN: LA BÚSQUEDA DE LA VERDAD
}

\author{
Biviana Unger Parra* \\ doi:10.11144/Javeriana.uph33-67.acbv
}

\begin{abstract}
RESUMEN
La influencia del Arpinate en el desarrollo vital e intelectual de Agustín es indiscutible. Existen varios estudios que rastrean la relación entre los dos pensadores, en particular, en lo que concierne a la retórica clásica y a la recepción agustiniana de las categorías de Cicerón. Sin embargo, hay un aspecto crucial que no ha sido abordado aún de manera exhaustiva, se trata de la relación del joven Agustín con el veterano Cicerón, esto es, la relación entre dos compositores de diálogos filosóficos que, hasta ese momento, se habían ocupado de la retórica. La intención aquí es la de rastrear dicha relación a partir de un diálogo que, por su carácter, nos ofrece una posición hermenéutica privilegiada: el Contra Academicos.

Palabras clave: Contra Academicos; San Agustín; diálogos; Cicerón; verdad
\end{abstract}

* Pontificia Universidad Javeriana, Bogotá, Colombia.

Correo electrónico: bivianaunger@gmail.com

Para citar este artículo: Unger Parra, B. (2016). Agustín y Cicerón: la búsqueda de la verdad. Universitas Philosophica, 33(67), pp. 201-215. ISSN 0120-5323, ISSN en línea: 2346-2426, doi:10.11144/Javeriana.uph33-67.acbv 


\title{
AUGUSTINE AND CICERO: THE SEARCH FOR TRUTH
}

\author{
Biviana Unger Parra
}

\begin{abstract}
Cicero's influence in the vital and intellectual development of Augustine is indisputable. There are several studies that track the relationship between the two thinkers, in particular, as far as classical rhetoric and the Augustinian reception of Cicero's categories concerned. However, there is a crucial aspect that has not yet been comprehensively addressed, it is the relationship of the young Augustine with veteran Cicero, that is, the relationship between two composers of philosophical dialogues which, until then, had dealt with rhetoric. The intention here is to trace this relationship from a dialogue which, by its nature, gives us a privileged hermeneutical position: the Contra Academicos.
\end{abstract}

Key words: Contra Academicos; St. Augustine; dialogues; Cicero; truth 
¿CuÁl es la Razón por la CUAl los estudiosos no han dedicado suficiente atención a la relación de Agustín con la filosofía ciceroniana? Uno de los motivos tiene que ver con el problema que el pensamiento mismo de Cicerón suscita, pues la relevancia filosófica de su obra ha sido objeto de un largo debate en el que es posible identificar dos posiciones. La primera considera que Cicerón se limita a presentar y recoger las ideas de diferentes escuelas filosóficas, sin profundizar en ninguna de ellas, asumiendo una posición ecléctica. La segunda, en cambio, ve en el Arpinate a un pensador original que, si bien se apropia de la tradición, lleva a cabo un trabajo único y novedoso que logra introducir a la filosofía en el mundo romano ${ }^{1}$.

La intención en este texto es la de examinar la influencia de Cicerón en el pensamiento del joven Agustín, en particular en el diálogo Contra Academicos. En primer lugar, se hará un breve recorrido por los diálogos de juventud de Agustín, de los cuales se ofrecerá una propuesta de lectura que defiende su unidad y que está organizada según un principio presente en la filosofía de Cicerón. En segundo lugar, se abordará la cuestión del escepticismo, con el fin de entender el papel que cumple el pensador romano en el Contra Academicos de San Agustín.

\section{Situación de los diálogos de Casicíaco en el desarrollo vital e intelectual de San Agustín}

En EL AÑo 386 Agustín ABANDona su cargo de orador de la corte de Milán y decide retirarse a la villa campestre de su amigo Verecundo, ubicada no muy lejos de la capital del Imperio. Su intención es la de formar una comunidad cristiana dedicada al estudio y a la oración, tomando distancia de las congojas del siglo y preparando el alma para el bautismo.

1 El punto de quiebre en la discusión se da gracias a la publicación de la impresionante obra Cicero Academicus de Carlos Lévy, en la que el autor recorre toda la historia de la Nueva Academia y de sus principales exponentes y hace un estudio exhaustivo de los diálogos ciceronianos, su estructura y fuentes.

Son de fundamental importancia los estudios de M. Testard (1958) y H. Hagendhal (1967) en los cuales se rastrea la relación entre Cicerón y Agustín, a partir de un cuidadoso estudio de la presencia de motivos ciceronianos en la obra del Hiponate. 
Las conversaciones con la madre, los amigos y el pequeño Adeodato son la fuente principal de inspiración para la intensa producción escrita realizada en este periodo, que se hará en su totalidad bajo la forma del diálogo.

Casicíaco marca para Agustín la etapa en la que construye las respuestas a muchas de las preguntas de su juventud; es el momento de recorrer los propios pasos y de ajustar cuentas con las diferentes fuentes de las que hasta el momento había bebido. Es así que surge la exigencia de ordenar lo aprendido. De ahí, el proyecto de una cultura cristiana sobre las disciplinas y las artes liberales pero, también, la dedicada y ardua composición de los diálogos: De beata vita, Contra Academicos y De ordine. La influencia de Cicerón en estas tres obras es fundamental, pues no solo se hace una constante alusión explícita a la autoridad del maestro romano, sino que los problemas filosóficos tratados remiten a diálogos ciceronianos específicos, y constituyen una revisión y respuesta a las propuestas del Arpinate. Así, podríamos decir que el De beata vita retoma y revisa algunas de las propuestas de Cicerón en torno a la cuestión de la felicidad y la virtud en las Tusculanae Disputationes; el Contra Academicos es una clara respuesta a los Academici libri y, por último, el De ordine recoge algunos temas fundamentales desarrollados en el De natura deorum y el De fato ${ }^{2}$.

\section{Unidad de los diálogos de Casicíaco: una propuesta de lectura}

PARA ENTENDER MEJOR LA MANERA COMO SE DA LA RELACión entre estas obras, podemos recurrir a un principio fundamental de la ontología agustiniana, a saber, la terna modus, species, ordo, a la cual Agustín hará alusión explícita en textos posteriores. Esta terna proviene de la retórica clásica, y la familiaridad de Agustín con la misma puede tener que ver con su amplio conocimiento de Cicerón, quien la menciona expresamente en el libro primero del De officiis ${ }^{3}$, último de los diálogos filosóficos del Arpinate. En los primeros diálogos agustinianos asistimos a una apropiación y reinterpretación de la terna ciceroniana en clave estético-ontológica, además de cumplir una función hermenéutica. La lectura

2 Véase: Foley, 2009.

3 Véase: Cicerón, 2001, I, 15. 
propuesta consiste, entonces, en establecer una relación entre los tres primeros diálogos y cada uno de los elementos de la terna.

De esta forma, el De beata vita respondería a la exigencia vital y metodológica de establecer un principio ordenador de la constitución misma del ser, el mo$d u s$, que en cuanto medida implica una idea de perfección y de plenitud. En el primero de los diálogos, la alusión al modus aparece con ocasión de la reflexión acerca de la virtud y, retomando una idea ciceroniana, se sostiene que "las cosas excesivas necesitan medida o modo: luego la abundancia supone cierta pobreza, mientras la medida excluye lo excesivo y lo defectuoso" (b.vita., 4. 32). Si bien, como ya lo afirmamos antes, el contexto original de la noción de modus es éticoretórico, asistimos aquí a una reinterpretación desde una perspectiva estéticoontológica, necesaria para poder responder a los numerosos interrogantes que estarán en la base del proyecto escrito agustiniano. ¿De dónde surge dicha lectura? ¿Cuáles son los pasos del camino de Agustín que determinan esta visión? Estas son solo algunas de las preguntas que quedan abiertas en la inagotable investigación de la obra agustiniana, ya que su resolución supone un estudio detallado de su paso por el maniqueísmo y los años anteriores a su conversión.

Una vez establecido el modus, se pasa a considerar el objeto mismo de la investigación, la species, esto es, la forma, el sello distintivo de la verdad de los seres. De la forma, en este sentido, se tratará en el Contra Academicos, donde se establece con claridad la diferencia entre la hermosura de las cosas sensibles y la verdadera Hermosura, hacia la cual deben estar dirigidos los pasos del caminante, y cómo la primera debe ser superada por la segunda. Dicho ascenso no implica una superación negativa, pues los grados a través de los cuales se llega a la verdadera Belleza hacen parte de un camino que todos los hombres deben recorrer en su calidad de seres racionales. Esta concepción se basa en el principio de que le pertenece únicamente a la razón la facultad de contemplar la pura belleza inaccesible a los sentidos, y a la razón compete, por tanto, llevar a cabo la racionalización de los mismos.

Por último, el diálogo De ordine, tanto en su forma como en su contenido, representa el esfuerzo por hacer evidente el principio estético que determina toda la naturaleza: el ordo. En este diálogo, se muestra sin reserva que el orden se manifiesta en todas las esferas de la creación y que, por este motivo, nadie es insensible ante la acción de la belleza. El mundo es un mundo ordenado pues, luego de establecer la materia, Dios le da forma a las cosas y las dispone ordenadamente 
creando así las relaciones que permitirán el funcionamiento de todo el conjunto. El mundo, al igual que un mosaico, es ordenado en virtud de la unidad y debe ser juzgado según el conjunto y no según los detalles considerados en sí mismos, ya que este modo de mirar las cosas se asemeja al del que restringiendo el campo visual y abarcando con sus ojos sólo el módulo de un azulejo de un mosaico, censurara al artífice como ignorante de la ordenación y composición de tales obras; creería que no hay orden en la combinación de las teselas, por no considerar ni examinar el conjunto de todos los adornos que concurren a la formación de una faz hermosa. (Agustín, 1994, 1. 1.2)

El mundo contemplado de manera correcta se muestra armónico, ordenado y hermoso; sin embargo, aquellos que no saben mirar serán incapaces de comprender dicho orden. Es necesario, entonces, disponer el ánimo de manera tal que puedan llegar a comprender cómo Dios gobierna todo con orden. ¿Cómo lograr esto? ¿Cuál camino debe seguirse para llegar a entender algo que está en aparente contradicción con aquello que muestra la experiencia? En varios pasajes del De ordine, Agustín hace alusión a la necesidad de preparar y purificar el alma a través de un arduo trabajo intelectual, que comienza con las artes liberales y termina con el conocimiento de las cosas mismas. Llama la atención el modo como Agustín exhorta a su joven interlocutor Licencio para que se dedique a la poesía, indicándole que, gracias a la práctica de las artes liberales, el alma se cura y se vuelve más ágil, pura, bella y apta para la contemplación de la verdad.

Podemos decir así que, en los diálogos de juventud, se recogen algunos problemas fundamentales, organizándolos según un esquema tripartito de origen ciceroniano basado en la terna modus, species, ordo, y usando como hilo conductor algunas categorías que serán fundamentales a lo largo del camino vital e intelectual de Agustín, a saber, veritas, caritas y pulchritudo.

3. Los diálogos de Casicíaco como respuesta a los diálogos ciceronianos: el Contra Academicos y las Cuestiones académicas

La Relación entre Agustín y Cicerón suele centrarse en el famoso episodio narrado en las Confessiones donde leemos que el joven estudiante de retórica, durante su permanencia en Cartago, siguiendo el orden de sus estudios, llega al Hortensius, muy aclamado por su estilo, mas no por su contenido que, al parecer, 
pasaba desapercibido para los estudiantes de elocuencia preocupados solo por mejorar su técnica. ¿Cuál es la reacción que produce el Hortensius? ¿Qué se encuentra en esta obra que determinará un cambio tan profundo en su lector? Consideremos este pasaje:

Semejante libro cambió mis afectos y mudó hacia ti, Señor, mis súplicas e hizo que mis votos y deseos fueran otros. De repente apareció a mis ojos, vil toda esperanza vana, y con increíble ardor de mi corazón suspiraba por la inmortalidad de la sabiduría, y comencé a levantarme para volver a ti. Porque no era para pulir el estilo - que es lo que parecía debía comprar yo con los dineros maternos en aquella edad de mis diecinueve años, haciendo dos que había muerto mi padre-; no era, repito, para pulir el estilo para lo que yo empleaba la lectura de aquel libro, ni era la elocución lo que a ella me incitaba, sino lo que decía.

¡Cómo ardía, Dios mío, cómo ardía en deseos de remontar el vuelo de las cosas terrenas hacia ti, sin que yo supiera lo que entonces tú obrabas en mí! Porque en ti está la sabiduría. Y el amor a la sabiduría tiene un nombre en griego, que se dice filosofía, al cual me encendían aquellas páginas. (Agustín, 1946, III, 7-8)

El camino hacia la sabiduría que inicia con la lectura del Hortensius estará lleno de obstáculos y laberintos, en uno de los cuales, el maniqueísmo, Agustín habría de permanecer perdido durante casi una década. Pero una vez más, una lectura se ofrece como luz y guía, se trata de los libros de los platónicos (platonicorum libri) gracias a los cuales Agustín abandona el materialismo maniqueo y encuentra la disposición adecuada para tratar aquellos problemas que la lectura del Hortensius había suscitado, los cuales hallarán concreción en Casicíaco. Es importante anotar que cuando la pequeña comunidad de cristianos se alberga en la Brianza, la intención es la de dedicarse al estudio de la filosofía y prepararse para recibir el bautismo. ¿Cuáles fueron las lecturas que acompañaron dicha preparación? La tradición, por mucho tiempo aceptada, sostenida por Alfaric, ha querido poner el énfasis en la comprensión de la vida de Agustín como una cadena de conversiones: maniqueísmo, escepticismo, platonismo y, por último, cristianismo. Apoyar esta postura no solo supone una visión muy restringida del desarrollo vital e intelectual de nuestro autor, sino una concepción de la construcción de su pensamiento fragmentada y pobre. Cuando Agustín se reúne en Casicíaco, 
seguramente tenía a la mano algunos de los libros platónicos que circulaban en el inquieto ambiente milanés pero, también, sin duda, consultaba los textos de quien había sido su maestro y modelo desde los diecinueve años: Cicerón.

Para apoyar esta idea pasamos a considerar el Contra Academicos, el más extenso y complejo de los diálogos de este periodo. Desde las primeras páginas de la obra, en la dedicatoria a Romaniano, encontramos una declaración que suele pasar desapercibida, con respecto a la preparación de los acompañantes de Agustín en Casicíaco, los jóvenes Licencio y Trigecio, a propósito de los cuales se afirma: "al verlos mucho más dispuestos y ansiosos de lo que yo me había imaginado, intenté entonces probar de qué eran capaces a su edad; porque me parecía que un libro de Cicerón, el Hortensius, les había ganado en gran medida para la filosofía" (Agustín, 1994, I, 1.4). Esta afirmación nos ofrece un dato fundamental: Agustín se apoya en su propia experiencia vital, y tal como el diálogo de Cicerón acerca de la búsqueda de la sabiduría había tenido para su vida un valor propedéutico, así mismo lo tendría para todos aquellos que quisieran dedicar su vida a la búsqueda de la verdad. El Hortenisus es, entonces, el punto de partida, no solo de este diálogo, sino del amplio proyecto agustiniano, por cuanto su contenido dispone a sus lectores hacia el camino recto que corresponde a la búsqueda de la sabiduría y la verdad. Así pues, tanto la influencia de las lecturas literarias y filosóficas que acompañaron a Agustín durante toda su vida, como la elección de una vida en comunidad alejada de los placeres mundanos y dedicada al enriquecimiento espiritual, inspirada en la tradición del otium liberale, le permitieron encontrar un campo de actividad intelectual del cual ya nunca se apartaría.

El punto de partida de dicha actividad es la afirmación, en contra del escepticismo, de que sí es posible encontrar la verdad. Agustín había encontrado en las lecturas platónicas una ontología de la espiritualidad que le permitió resolver algunas cuestiones que siempre habían estado en el centro de sus reflexiones, a saber, la naturaleza de Dios y el problema del mal. La incorrecta ontología maniquea es remplazada ahora y puesta al servicio de la fe; esto no puede menos que entusiasmar a Agustín que, consciente del error maniqueo, buscaba desesperadamente respuestas a sus interrogantes. Debemos concluir entonces que de las lecturas platónicas se tomaron ciertos elementos y se desecharon otros, no compatibles con el cristianismo, adoptando así una postura crítica que queda claramente evidenciada en el rechazo hacia la posición escéptica de la Nueva 
Academia, la cual se declaraba a sí misma heredera del pensamiento platónico. ¿Es el Contra Academicos una respuesta al escepticismo ciceroniano? Para responder a este interrogante debemos entender la naturaleza del diálogo ciceroniano Cuestiones Académicas y la postura escéptica en ellos expuesta.

Cicerón escribe el diálogo Cuestiones académicas en un momento muy fructífero de su producción filosófica, cuando se aleja de los avatares de la vida pública y se retira a su villa de Túsculo para dedicarse de lleno a la filosofía. Las dos primeras obras de este periodo, comprendido entre el 45 a.C y el 43 a.C, tienen un carácter propedéutico y exhortativo, pues se trata de la Consolatio y el Hortensius, ambos perdidos. La tercera obra compuesta es Cuestiones Académicas, de la que conservamos incompleto el primer libro de la tercera edición, conocido como Academica posteriora I, y el segundo libro de la primera redacción conocido como Lucullus o Academica priora II. Hablamos de diferentes ediciones porque durante su composición el autor realizó varios cambios, tanto en la estructura de la obra, de dos libros pasó a cuatro, como en los personajes de la misma. Esto último no debe entenderse como un mero dato anecdótico. La elección de los personajes de sus diálogos estaba estrechamente ligada con su intención de llevar la filosofía a las más altas esferas del mundo romano, por este motivo, elegía cuidadosamente a sus interlocutores como bien podemos constatarlo en las cartas a Ático, quien le aconsejó algunos cambios en el contenido y la dedicatoria. Aunque no podemos saber el contenido de las partes perdidas, sabemos que trataba de la exposición de las posturas de Cárneades y de Antíoco y las refutaciones de cada una de ellas. En lo que conservamos encontramos la disputa de Antíoco de Ascalona en contra de Arcesislao y Cárneades, en lo que concierne a su teoría del conocimiento ${ }^{4}$. Antíoco, discípulo de Filón, había seguido las propuestas de los fundadores de la Nueva Academia, Arcesislao y Cárneades, durante gran parte de su vida aunque en sus últimos años abrazó el estoicismo y sostuvo que los estoicos eran los verdaderos continuadores de la tradición platónica de la Academia, alejándose así del escepticismo neo académico que proponía una actitud anti dogmática basada en la suspensión del juicio y en la

4 Arcesislao (315-249 a.C), fundador de la Nueva Academia, rechazó el principio estoico del asentimiento, $\sigma \nu \gamma \alpha \tau \tau \dot{\theta} \theta \sigma \iota \zeta$, según el cual se puede garantizar la absoluta veracidad de las sensaciones cuando el alma, frente a una representación cataléptica, la aprueba como verdadera, en cuanto le resulta evidente. 
probabilidad. ¿Cuál es la posición que asume Cicerón en esta disputa y cuál es su intención al exponer la crítica de Filón? Para responder a estos interrogantes debemos preguntarnos cuál fue la posición de Cicerón respecto al problema de la verdad. En primer lugar, debemos distinguir que en el escepticismo promulgado por dos de los más importantes representantes de la Nueva Academia hay una discrepancia, pues mientras Arcesislao proponía un escepticismo radical, Cárneades, recurriendo a la probabilidad, representa una vertiente moderada en la propuesta escéptica. Si nos atenemos a la declaración explícita de Cicerón en el De natura deorum, la respuesta es clara:

debido a que nosotros no nos encontramos en la posición de negar la existencia de la verdad, sino de decir que cada verdad viene acompañada del error, debemos admitir que éste es tan similar a aquélla, que no podemos darle nuestro asentimiento. De esto deriva que muchos conocimientos son probables y aunque no los entendamos, gobiernan la vida del sabio porque presentan un aspecto notable e ilustre. (Cicerón, 1984, 1, 12)

Tenemos entonces que, si bien Cicerón se considera el último representante de la Nueva Academia y gran admirador de Cárneades, como él mismo lo afirma unas líneas después del anterior pasaje ${ }^{5}$, es claro que su academicismo reinterpreta las nociones de 'suspensión del juicio' (epoché) y 'probabilidad' (pithanon) que habían sostenido sus predecesores, proponiendo una búsqueda de la verdad anti dogmática y en continua transformación. La acusación inmediata frente a una propuesta de este tipo es la de relativismo moral, que podemos recogerla recurriendo a las palabras que Lúculo le dirige a Cicerón en las Cuestiones Académicas: después de que tributaste (en el Hortensio) tan grandes alabanzas a la filosofía, ¿seguirás esas doctrinas que todo lo confunden, que nos despojan del juicio, del asentimiento y de los sentidos? Después de haber dicho, bajo juramento, que tú estabas seguro de aquellas ocultas maquinaciones (las de Catilina), ¿vas a decir ahora que nada se puede conocer o percibir? (Cicerón, 1990, 61-62)

La auto ironía ciceroniana del anterior pasaje no es casual. El autor del diálogo sobre las Cuestiones Académicas expone su pensamiento a través de todos los personajes que utiliza y no solo en el que lleva su nombre. Cicerón buscó la verdad toda su vida, no solo en los tribunales y la vida pública romana, sino en sus 
escritos, sobre todo en aquellos dedicados a la filosofía, compuestos en los últimos tres años de su vida ${ }^{6}$. Si admitimos esto, podemos entender uno de los pasajes más discutidos del Contra Academicos de Agustín en el que se afirma:

Para que los razonamientos de los académicos no parezcan extender como ciertas nieblas, ni les parezca a algunos que nos oponemos enérgicamente a la autoridad de muy doctos varones, y especialmente a la de Tulio Cicerón, quien no puede dejarnos indiferentes, haré antes un breve comentario, si os parece, oponiéndome a quienes creen que sus enseñanzas van contra la verdad. (Agustín, 2009, 3, 7, 14)

En este punto, debemos preguntarnos con Giovanni Catapano (2006): “¿qué tipo de escepticismo critica Agustín en el Contra Academicos?". Sin duda aquel que niega la posibilidad de encontrar la verdad, pues en su búsqueda ha puesto innumerables esfuerzos desde que el Hortensius lo incitó a tomar el camino de la filosofía, así como también lo hizo Cicerón en sus obras y en su vida. Tanto para Agustín, como para Cicerón, la filosofía "en cuanto inquisitio veri es una empresa intelectual y existencial, que requiere una fuerte motivación interior, motivación que el escepticismo académico, en la medida en que conduce a la desperatio veri, podría destruir" (Catapano, 2006). Pero entonces ¿cómo explicamos el escepticismo de Cicerón y la afinidad de Agustín con este último?

Acerca de esto, resulta iluminadora la propuesta de Marchand según la cual, Agustín reinterpreta el escepticismo entendiéndolo como un momento crítico previo a la filosofía y posibilitador de su práctica ${ }^{7}$. De hecho, en el último libro del Contra Academicos y en la Carta a Hermogeniano, Agustín propone una hipótesis, muy controvertida ${ }^{8}$ acerca del carácter esotérico de la Nueva Academia. Consideremos la Carta a Hermogeniano:

6 Un aporte fundamental al estudio del carácter filosófico de las cartas de Cicerón, lo debemos a Mc Connell (2014).

7 Véase: Marchand, 2013.

8 Charles Brittain es uno de los opositores más fuertes de la hipótesis presentada por Agustín. Afirma que este último "ofrece una increíble e implausible teoría de la historia griega desde Sócrates hasta Plotino, a saber, la teoría del esoterismo platónico en la Academia escéptica e interpreta los objetivos filosóficos de Cicerón en las Cuestiones Académicas, a partir de esta hipótesis" (Brittain, 2000, p. 85ss). 
Nunca me hubiera yo permitido, ni aun en broma, la osadía de combatir a los académicos (¿cómo no iba a impresionarme la autoridad de tan graves varones?), si no fuese porque estimo que su doctrina es muy otra de la que el vulgo les atribuye. Mejor que refutarlos, lo que no puedo, traté de imitarles como podía. Pienso que en aquel tiempo era más oportuno encauzar la corriente platónica, si algo manaba puro de su fuente, por un boscaje sombrío y espinoso a disposición de unos pocos, que dejarla correr a campo abierto ante los rebaños que aparecen a cada momento e imposibilitan el conservarla pura y limpia. ¿No es lo más conveniente para una bestia opinar que el alma es corporal? Pienso, pues, que contra esta gente se urdió útilmente el arte y razón de ocultar la verdad. (Agustín, 1951, 1)

Según Agustín, el escepticismo de la Nueva Academia nace en contraposición al materialismo estoico, es incapaz de comprender las verdades metafísicas del platonismo, y se opone al dogmatismo característico del estoicismo y el epicureísmo. Frente a la fuerza con la que estas escuelas se imponían, la única salida para salvaguardar la verdad, esto es, las verdades socráticas, parecía ser su ocultamiento. Por el momento, dejemos de lado la veracidad histórica de esta hipótesis presentada por Agustín y centrémonos en su utilidad. Para hacerlo, debemos tener en cuenta, en primer lugar, el papel que tuvo el escepticismo, como propuesta filosófica en el desarrollo vital e intelectual del joven Agustín. Sabemos que la lectura del Hortensius había logrado su cometido logrando que el joven estudiante de retórica se llenara de deseos por dedicarse a la búsqueda de la verdad. Como ya lo afirmamos, el camino no fue fácil y el materialismo y el dogmatismo maniqueos se presentaron seductores proponiendo falsedades como verdades absolutas. Frente a la desilusión causada por el maniqueísmo y su incapacidad para responder a las más agudas cuestiones, Agustín entró en un periodo que él mismo llamó escéptico. Veamos:

Sin embargo, considerando y comparando más y más lo que los filósofos habían sentido acerca del ser físico de este mundo y de toda la Naturaleza, que es objeto del sentido de la carne, juzgaba que eran mucho más probables las doctrinas de éstos que no las de aquéllos [maniqueos]. Así que, dudando de todas las cosas y fluctuando entre todas, según costumbre de los académicos, como se cree, determiné abandonar a los maniqueos, juzgando que durante el tiempo de mi duda no debía permanecer en aquella secta, a la que anteponía ya algunos filósofos, a quienes, sin embargo, no quería encomendar de 
ningún modo la curación de las lacerías de mi alma por no hallarse en ellos el nombre saludable de Cristo. (Agustín, 1946, 5, 14, 25)

Con los elementos recogidos hasta ahora podemos afirmar que la actitud de Agustín frente al escepticismo no es negativa, por cuanto esta se constituye como momento preparatorio para el encuentro con la verdad. Esto nos lleva a distinguir entre dos tipos de escepticismo, a saber, uno radical, que se vuelve dogmático, y otro, moderado, que se presenta como estrategia en la búsqueda de la verdad. Ahora, queda todavía abierta la cuestión de cómo se relacionan Cicerón y Agustín con estos dos tipos de escepticismo que hemos descrito. En cuanto al orador romano, con Powell (2002, p. 23) sostenemos que su escepticismo probabilista puede considerarse como una estrategia realista frente al materialismo epicúreo y al dogmatismo estoico. De ser esto así, tendríamos que el escepticismo, en cuanto estrategia o actitud, cumple el mismo papel propedéutico en ambos pensadores. Pero, entonces, ¿cómo logra Agustín desprenderse del error maniqueo y sus promesas de alcanzar la verdad a través de un racionalismo materialista y dogmático? Haciendo suyo un principio de su maestro Cicerón, que afirma: "mientras que los demás dicen que unas cosas son ciertas y otras son inciertas, yo, disintiendo de ellos, digo que unas cosas son probables y otras improbables" (Cicerón, 1989, 2, 1, 7). El probabilismo ciceroniano no es, según como sostenemos que lo lee Agustín, un escepticismo radical que niegue al sabio la posibilidad de alcanzar la verdad, sino que, en cambio, funciona como una advertencia frente a la posibilidad latente de caer y mantenerse en el error. Por esta razón, en la parte final del Contra Academicos Agustín afirma:

$\mathrm{Al}$ notar yo por los gestos de la cara que los muchachos se mostraban un poco decepcionados, porque Alipio, al parecer, no iba a responder, les dije sonriendo:

- ¿Tenéis acaso envidia de las alabanzas que me ha tributado? Mas por ser tan segura la firmeza de Alipio, no le temo, y para que vosotros me mostréis también vuestro agradecimiento, quiero prepararos contra él, por haber defraudado vuestra esperanza. Leed los libros de los Académicos, y cuando veáis allí a Cicerón vencedor de estas bagatelas-¿̇y qué cosa más fácil que lograr esto?-, obligad a Alipio a sostener mi causa y razonamiento contra aquellos argumentos invencibles de Tulio, (Agustín, 1994, 3. 20. 45) 
¿De qué manera aparece Cicerón como vencedor? ¿Cuáles son sus argumentos invencibles y contra quién están dirigidos? Podríamos aventurarnos a decir que Agustín asume una interesante posición interpretativa que le permite ver los diálogos ciceronianos como el ejercicio dialéctico de un autor capaz de dar cuenta de las diferentes posiciones de las escuelas filosóficas de su tiempo. En este sentido, su victoria consistiría en mostrar su habilidad para defender las distintas posiciones, pero sin dejarse seducir por las falsas promesas del dogmatismo y manteniéndose firme en su búsqueda de la verdad. La réplica de Cicerón, con la que se inicia el cierre de los Academica posteriora podría entenderse desde esta perspectiva:

Cuando disputo con los demás, sobre todo acerca de cuestiones morales, no pretendo engañarlos a ellos ni tampoco a mí mismo, ni soy partidario de la obstinación o sofistería. Por lo cual, si fuera pertinente, juraría por Júpiter que tengo el mayor deseo de encontrar la verdad y que digo lo que pienso. Si me gozo al descubrir algo verosímil, ¿cómo puedo no ansiar descubrir lo verdadero? (Cicerón, 1990, 61)

Esa disposición hacia lo verdadero, entendida como amor a la sabiduría, fue el elemento que desde la lectura del Hortensius marcó la cercanía entre Agustín y Cicerón. ¿Cómo podría alguien exhortar a la filosofía si no cree posible encontrar la sabiduría? Que Cicerón haya escrito su diálogo académico justo después de sus dos obras exhortativas, no debe perderse de vista, pues podemos entenderlo como una advertencia, como un llamado a la prudencia y, en este sentido, en cuanto indicador en el camino hacia la verdad, Cicerón ha salido vencedor.

Bibliografía

Agustín de Hipona. (1951). Cartas. Madrid: BAC.

Agustín de Hipona. (1946). Confesiones. Madrid: BAC.

Agustín de Hipona. (1994). Contra los académicos. Escritos filosóficos. Madrid: BAC.

Agustín de Hipona. (1994). De la vida feliz. Escritos filosóficos. Madrid: BAC.

Brittain, C. (2000). Augustine as a Reader of Cicero. Tolle lege: Essays in Honor of Roland J. Teske. S.J. (pp. 81-114). Wisconsin: Marquette University Press. Brittain, C. ( 2006). Cicero, On Academic Scepticism. Indianapolis: Cambridge. 
Catapano, G. (2006). Quale scetticismo viene criticato da Sant' Agostino nel "Contra Academicos"? Quaestio, 6, pp. 1-13.

Cicerón, M.T, (1990). Cuestiones académicas. México D.F.: UNAM.

Cicerón, M.T. (1984). Sobre la naturaleza de los dioses. Madrid: Sarpe.

Cicerón, M.T. (1989). Sobre los deberes. Madrid: Tecnos.

Cicerón, M.T. (2010). Tusculanas. Madrid: Alianza.

Courcelle, P. (1968). Recherches sur les Confessions de Saint Augustin. Paris: Éditions E. de Boccard.

Foley, M. (1999).Cicero, Augustine and the Philosophical Roots of the Cassiciacum Dialogues. Revue des Études Augustiniennes, 45, pp. 51-77.

Hagendahl, H. (1967). Augustine and the Latin Classics. Göteborg: Acta Universitatis Gothoburgensis.

Lévy, C. (1992). Cicero Academicus. Roma: École française de Rome.

Malaspina, E. (2012-2013). Cicerone e la verità. Res publica litterarvm. Documentos de trabajo del grupo de investigación "Nomos" (pp. 1-18). Madrid: Instituto de Estudios Clásicos "Lucio Anneo Séneca”.

McConnell, S. (2014).PhilosophicalLife in Cicero'sLetters. Nueva York: Cambridge.

Marchand, S. (2013). Les Academica dans le Contra Academicos: détournement et usage du scpeticisme academicien par Saint Augustine. Astérion, 11 . https://asterion.revues.org/2336

Pollmann, K. \& Vessey, M. (2007). Augustine and the Disciplines. From Cassiciacum to Confessions. Nueva York: Oxford University Press.

Powell, J. (2002). Cicero the Philosopher. Twelve Papers. Nueva York: Oxford University Press.

Testard, M. (1958). Saint Augustin et Cicèron. Paris: Ètudes Agustiniennes.

Wills, B. (1999). Ancient Scepticism and the Contra Academicos of Saint Augustine. Animus, 4, pp. 108-123.

Woolf, R. (2015). Cicero. The Philosophy of a Roman Sceptic. Nueva York: Routledge. 